\title{
Microcavités à polymères du carbazole : vers des lasers plastiques bleus
}

\author{
C. Olivero, M.C. Castex, G. Pichler ${ }^{1}$, D. Adès ${ }^{2}$, E. Cloutet $^{2}$ et A. Siove ${ }^{2}$
}

\author{
Laboratoire de Physique des Lasers, CNRS, Université Paris Nord, 99 avenue J.B. Clément, \\ 93430 Villetaneuse, France \\ ${ }_{1}^{1}$ Institute of Physics, P.O. Box 304, 10000 Zagreb, Croatie \\ ${ }^{2}$ Laboratoire de Recherche sur les Macromolécules, CNRS, Université Paris Nord, \\ 99 avenue J.B. Clément, 93430 Villetaneuse, France
}

\begin{abstract}
Résumé : Nous présentons ici des résultats obtenus sur les propriétés de luminescence de polymères à base de carbazole excités par laser à $313 \mathrm{~nm}$. De nouvelles structures macromoléculaires ont été synthétisées dont les performances de luminescence sont comparables à celles de molécules modèles. Nous proposons, en outre, différentes solutions pour la réalisation d'une microcavité permettant de réaliser, à l'aide de ce type de matériaux, des lasers plastiques miniatures.
\end{abstract}

\section{INTRODUCTION}

L'enjeu de l'utilisation des propriétés optiques des matériaux polymères est considérable : ils associent les propriétés optiques de molécules organiques telles que les colorants, à la plasticité des polymères. En particulier, de grands efforts sont entrepris concernant la recherche de matériaux organiques dont la fluorescence est centrée sur les courtes longueurs d'ondes du spectre et plus précisément dans la région du bleu. Dans cette optique, la molécule de carbazole est un excellent candidat puisque dans sa conformation la plus simple, elle produit une émission bleue-violette intense. De plus, il a été montré [1] que par fonctionnalisation du carbazole par des substituants spécifiques, cette émission peut alors se décaler vers le rouge, offrant ainsi la possibilité d'obtenir les trois couleurs nécessaires aux afficheurs multicouleurs.

\section{LE POLYMERE EN ESCALIER}

Un polymère à espaceurs flexibles et à enchaînement rigide bicarbazyles a été élaboré. En plus de ce polymère à structure en escalier, nous disposons d'une molécule modèle, le dimère du $N$-éthyl carbazole. Celle-ci possède des propriétés optiques très intéressantes que nous avons déjà étudiées [2] mais possède des qualités filmogènes médiocres. Le but recherché avec le polymère est de disposer d'un matériau à propriétés optiques proches de celles du dimère mais en outre, avec de bien meilleures propriétés thermomécaniques et de processabilité. Il a été montré que c'est effectivement le cas. [3]

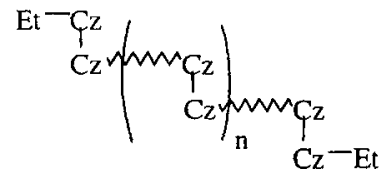

(a)

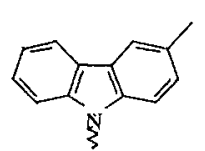

(b)

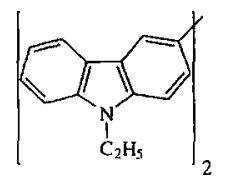

(c)

Figure 1: (a) Le polymère en escalier, (b) la molécule de carbazole, (c) le dimère du $N$-éthyl carbazole.

\section{EXPERIENCES DE PHOTOLUMINESCENCE}

L'objectif est de comparer les performances de luminescence du polymère à celles du dimère du $N$ éthyl carbazole, grâce à l'enregistrement des spectres d'émission de ces deux produits dans des conditions identiques. La source lumineuse utilisée est un laser à colorant doublé pompé par un laser Nd:YAG pulsé à $20 \mathrm{~Hz}$, l'ensemble générant en sortie des impulsions à $313 \mathrm{~nm}$ d'une durée de $7 \mathrm{~ns}$. Les échantillons étudiés sont sous forme de films ou de solutions. L'acquisition se fait à l'aide d'un matériel de type OMA et d'une caméra CCD intensifiée. Nos résultats montrent que le rendement de luminescence du polymère est comparable à celui du dimère et que les deux spectres sont identiques (fig. 2). 
Ainsi, on peut envisager d'utiliser, au même titre que le dimère, le polymère comme milieu actif dans un microlaser bleu.

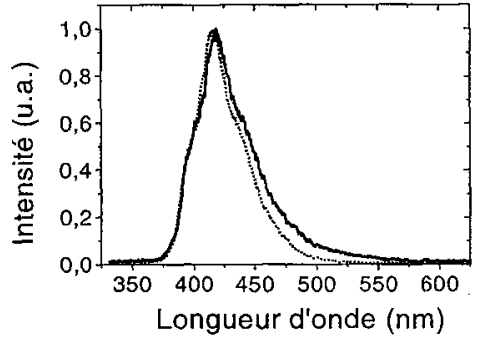

Figure 2 Spectres de photoluminescence du polymère en escalier $(-)$ et du dimère du $N$-éthyl carbazole (....) dans une solution de THF excitée à $313 \mathrm{~nm}$.

\section{VERS LA REALISATION DE MICROCAVITES}

Pour parvenir à l'obtention d'un effet laser, il est indispensable d'insérer le milieu actif dans une cavité. Mieux encore, nous voulons que celle-ci soit constituée par le milieu actif lui-même. La solution choisie consiste à réaliser une structure de type DFB (Distributed Feedback) en créant, à la surface du polymère d'indice $n$, un réseau de pas $\Lambda$ qui permet de sélectionner (à l'ordre $m$ près) une émission laser $\lambda=(2 \Lambda \mathrm{n}) / \mathrm{m}$. Nos premiers essais qui ont été réalisés à l'aide d'un masque de phase au contact d'échantillons de PMMA ou de PVC, ont conduit à des réseaux gravés micrométriques de bonne qualité (fig 3-a). Pour cela nous avons utilisé un laser à excimère émettant à $193 \mathrm{~nm}$. Cependant, l'élaboration d'un réseau de pas inférieur est souhaitable pour diminuer l'ordre $m$ et donc augmenter l'efficacité du processus. Une méthode interférométrique classique est à l'étude et devrait permettre de réaliser des réseaux sub-micrométriques. Enfin, une alternative intéressante consiste, à l'aide d'un seul faisceau incident, par interférence avec des ondes de surface, à créer des structures périodiques par le procédé dit de LIPSS (Laser Induced Periodic Surface Structure) [4]. Nous avons ainsi pu obtenir des structures périodiques de $140 \mathrm{~nm}$ environ (fig. 3-b).

(a)

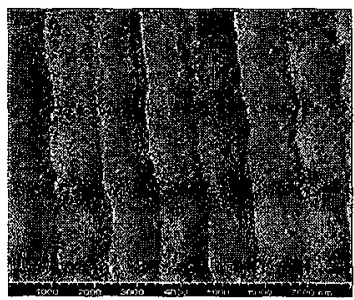

(b)

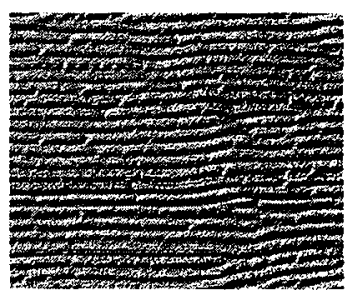

Figure 3 (a) Photo AFM d'un film de PVC structuré par masque de phase (pas $=1,2 \mu \mathrm{m}$ ) (b) Photo MEB d'un film de PET structuré par LIPSS (pas $=140 \mathrm{~nm}$ ). Dans les deux cas on utilise un laser ArF $(193 \mathrm{~nm})$.

\section{PERSPECTIVES}

L'ultime étape vers la réalisation d'un microlaser est de disposer d'un film homogène et suffisamment fin (quelques centaines de $\mathrm{nm}$ ) pour permettre le guidage des modes à l'intérieur de la cavité, de telle manière que la rétroaction optique apportée par le réseau, conduise à une émission stimulée à partir d'un seuil physiquement réalisable. Nos travaux s'orientent actuellement dans cette direction.

\section{Références}

1 D. Adès, V. Boucard, E. Cloutet, A. Siove, C. Olivero, M.C. Castex, G. Pichler, J. Appl. Phys. 87, $7290(2000)$

2. W.S Bacsa, M. Schaer, L. Zuppiroli, D. Adès, A. Siove, J. Appl. Phys. 84, 5733 (1998)

3 E. Cloutet, C. Olivero, D. Adès, M.C. Castex, A. Siove (à paraître)

4. S. Lazare, D. Drilhole - J. of Photochem. and Photobio. A, 106, 15 (1997) 